Supplementary Information for

\title{
Maximizing polyacrylonitrile nanofiber piezoelectric properties through optimization of electrospinning and post thermal treatment processes
}

Sooyoun Yua ${ }^{a}$ JoAnna Milam-Guerrero, ${ }^{a}$ Youyi Tai ${ }^{b}$, Steve Yang ${ }^{c}$, Yun Young Choi ${ }^{a}$, Jin Nam ${ }^{b}$, Nosang V. Myunga*

${ }^{a}$ Department of Chemical and Biomolecular Engineering, University of Notre Dame, Notre Dame, IN 46530, United States

${ }^{\mathrm{b}}$ Department of Bioengineering, University of California, Riverside, 900 University Ave. Riverside, CA 92521, United States

${ }^{c}$ Department of Materials Science and Engineering, University of California, Riverside, 900 University Ave. Riverside, CA 92521, United States

*Corresponding author E-mail: nmyung@nd.edu 

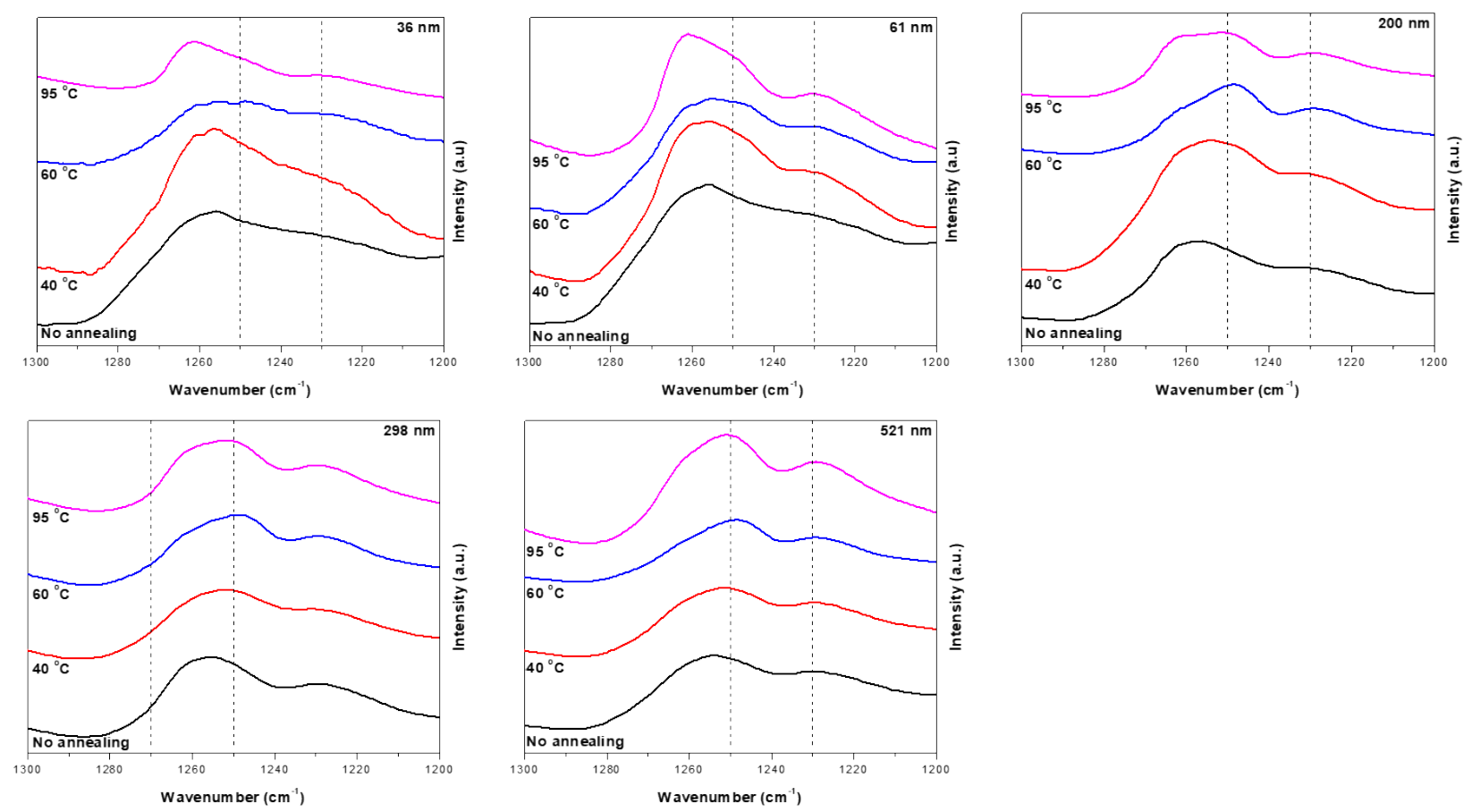

Figure S1. Annealing temperature-dependent FTIR for PAN nanofibers of various sizes. Diameters indicated at top-right corner of each plot are as-spun fiber size. 

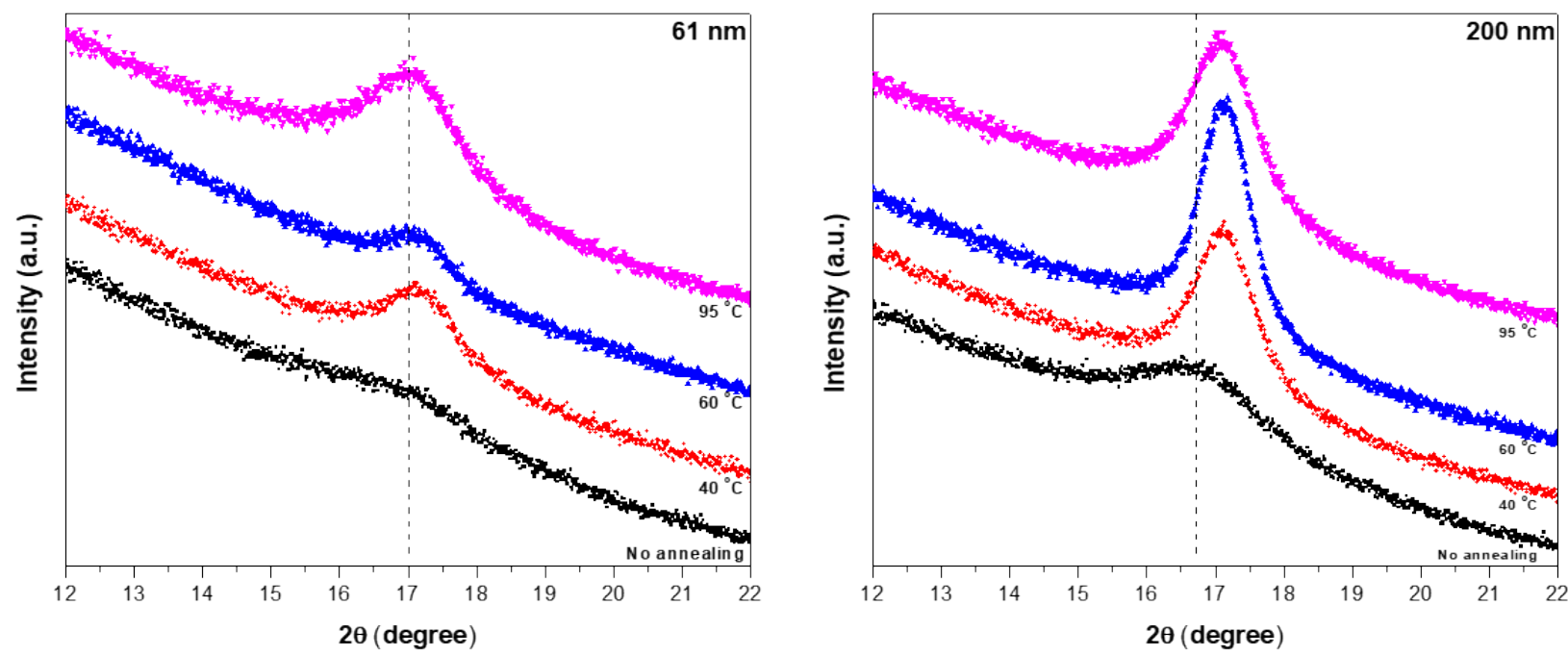

Figure S2. XRD spectra of PAN nanofibers annealed at various temperatures. Diameters indicated at top-right corner of each plot are as-spun fiber size. Dashed lines indicate peak location for asspun nanofibers (i.e. no annealing). 
(a)

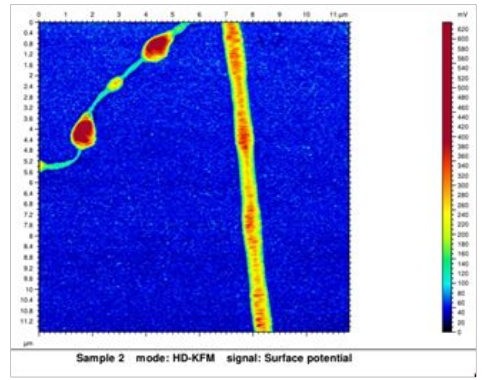

(b)

(d)

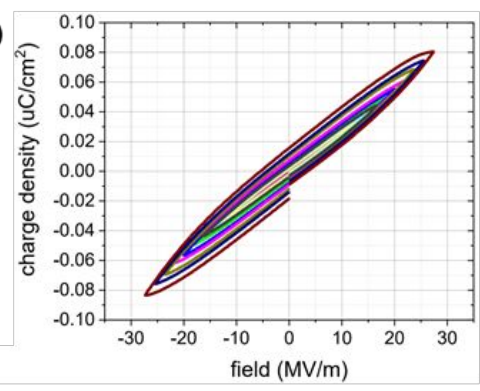

(f)

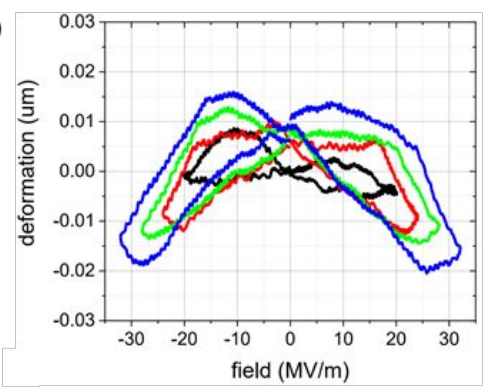

(c)

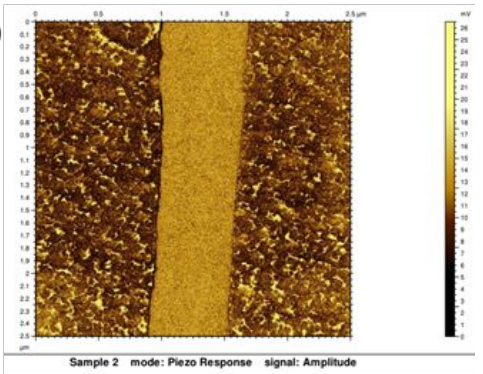

(e)

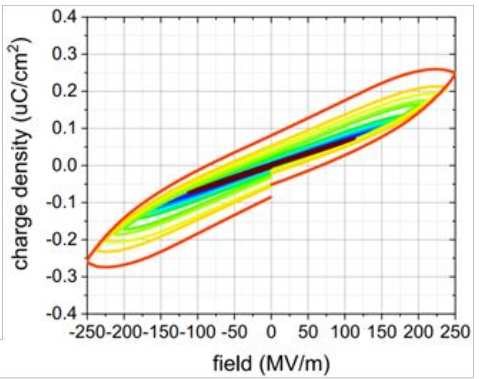

(g)

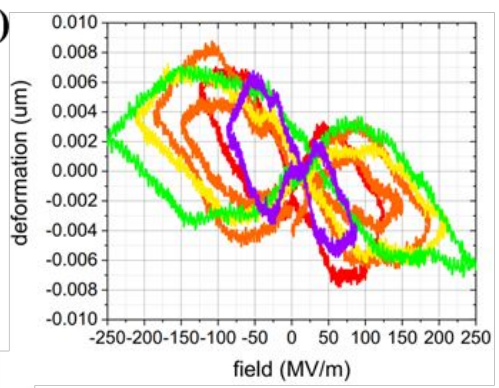

Figure S3. $(a, b)$ Color map showing the surface potential and (c) piezo response mapping across a single nanofiber. Ferroelectric loop of (d) as-spun 200-nm and (e) 61-nm PAN nanofiber annealed at $50^{\circ} \mathrm{C}$. Butterfly loop of (f) as-spun 200-nm and (g) 61-nm PAN nanofiber annealed at $50{ }^{\circ} \mathrm{C}$. 

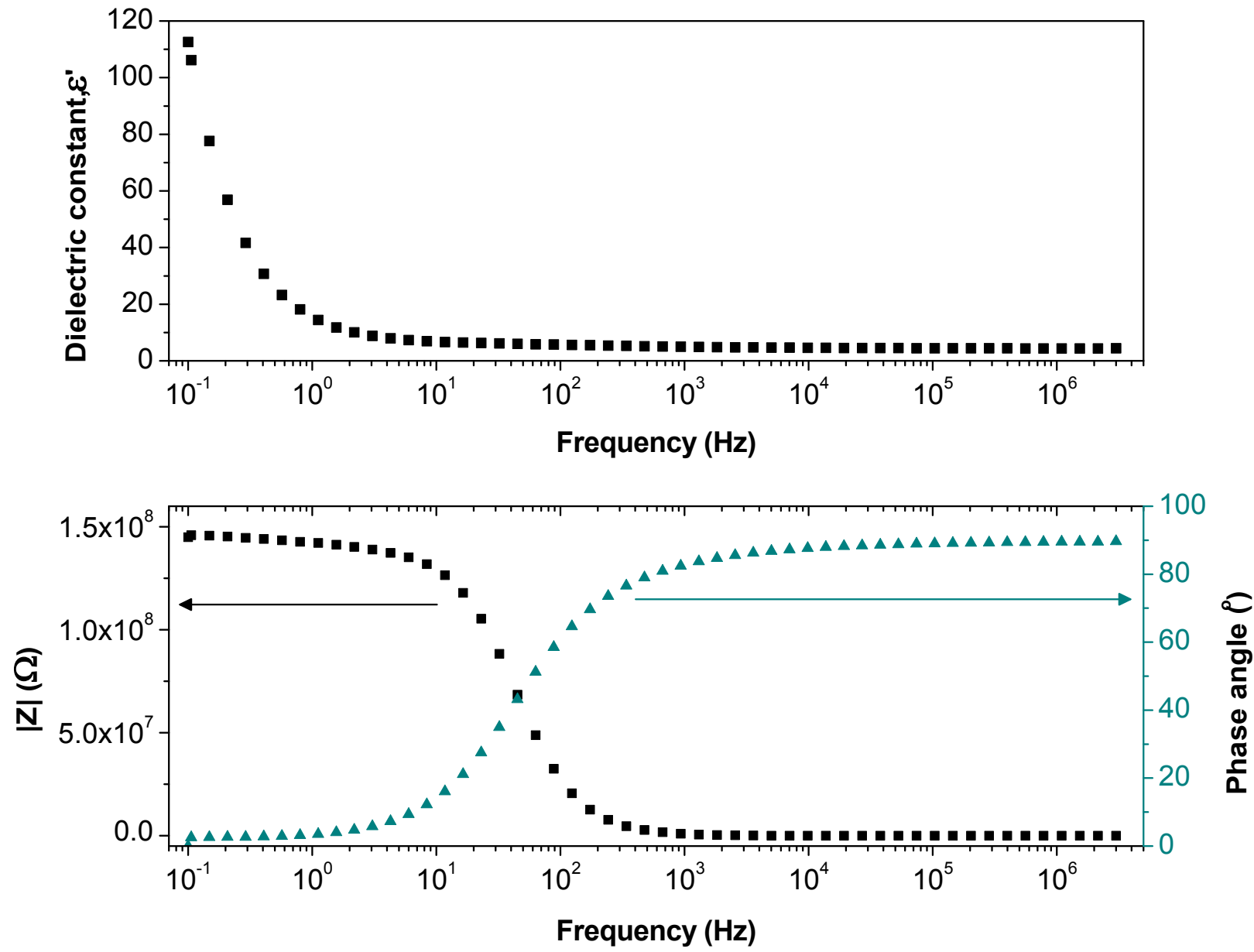

Figure S4. (a) Frequency-dependent dielectric constant and (b) Bode plot of as-spun PAN nanofibers. 


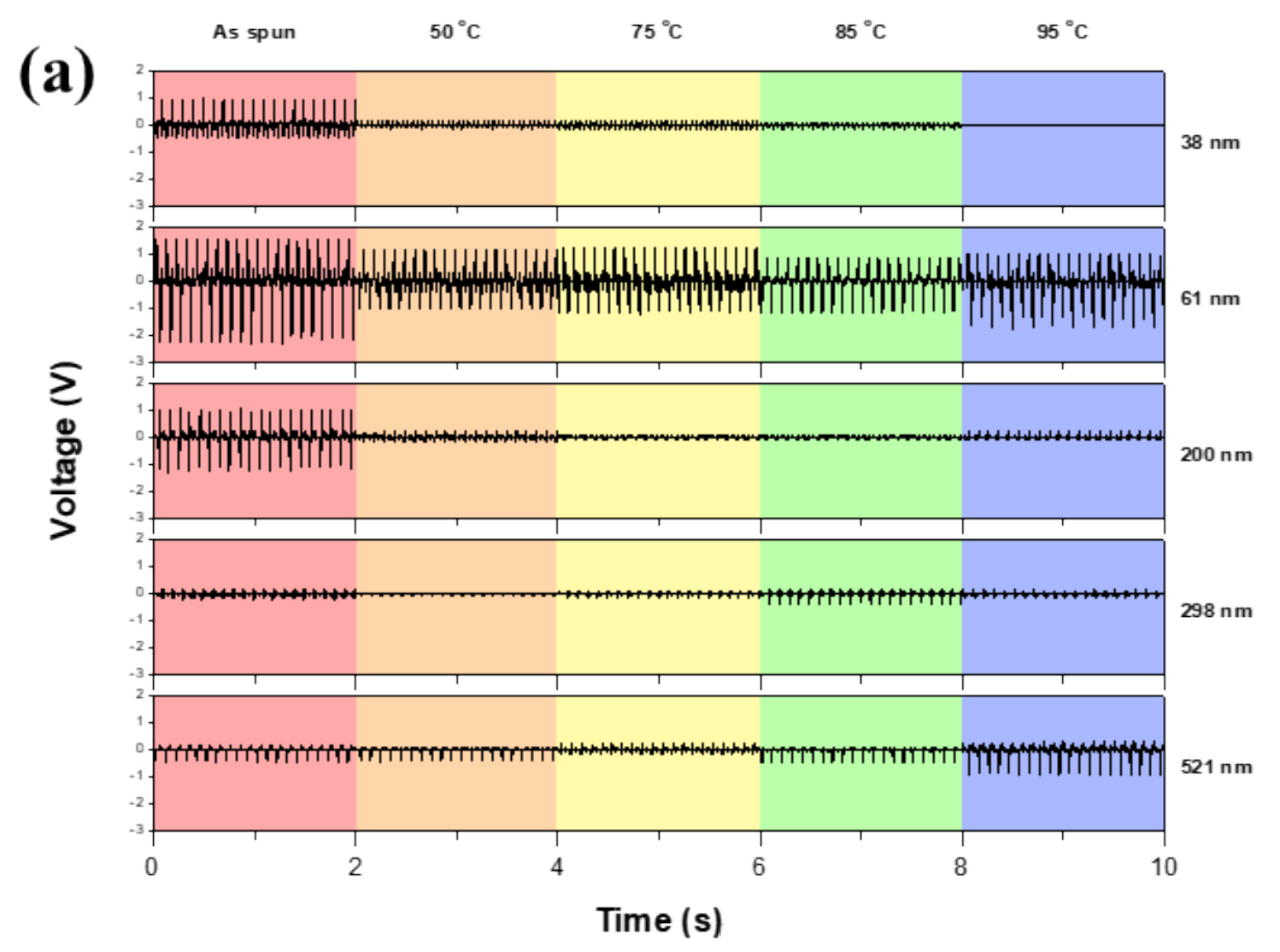

(b)

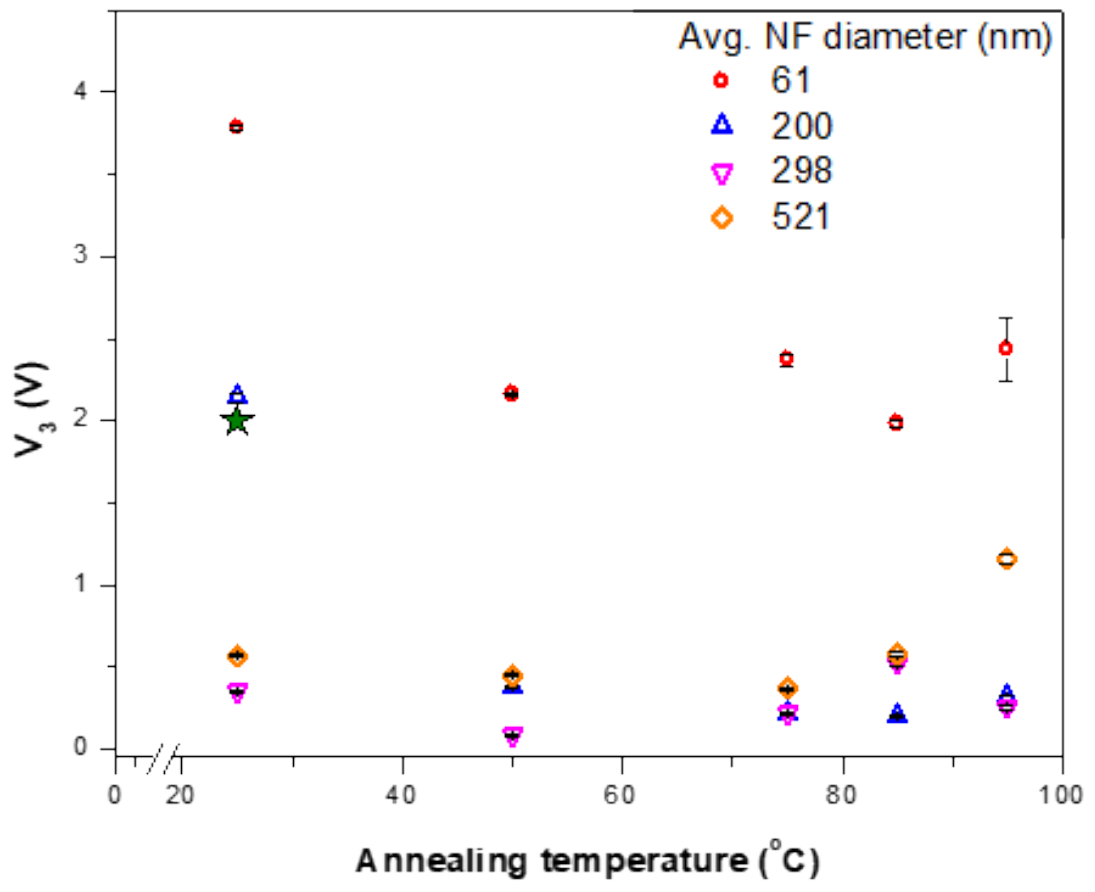

Figure S5. Size- and annealing temperature-dependent voltage response. (a) Raw data as a function of time and (b) scatter plot showing average peak-to-peak $\mathrm{V}_{3}$ as a function of annealing temperature for various average nanofiber diameter. Green star indicates literature value. 